\title{
Le principe de précaution
}

\author{
Precaution principle \\ par Pierre Delaporte \\ Président d'Espaces pour demain
}

The precaution principle was born in the 70's following the questions about the climatic modifications. If this principle is applied in a radical way, it leads to inaction. It therefore needs to be interpreted as a prudent way of acting (see Barnier's Law). A new approach of the decision reaching must be set up.

\section{L'ORIGINE ENVIRONNEMENTALE DU PRINCIPE DE PRÉCAUTION}

Le concept de précaution a émergé dans les années 70 en relation avec les interrogations sur le changement climatique (le trou dans la couche d'ozone, l'effet de serre : de nouvelles menaces pour l'avenir de la planète ?). Cette crainte exprimée par le corps social naissait de l'impuissance qu'il y aurait à revenir en arrière et de la relativité des savoirs scientifiques sur la question du changement climatique. Cette inquiétude appela alors une réaction politique tant au niveau international que national.

\subsection{Sur le plan international et européen}

Traduit juridiquement pour la première fois en 1985 dans la Convention de Vienne sur la protection de la couche d'ozone, le principe de précaution est entré dans le droit européen à l'article $130 \mathrm{R}$ du Traité de Maastricht (février 1992) qui le cite sans cependant le définir. Le principe 15 de la Déclaration de Rio sur l'environnement et le développement de juin 1992 le consacre en droit international en ces termes: “ pour protéger l'environnement, des mesures de précaution doivent être largement appliquées par les Etats selon leurs capacités. En cas de risques de dommages graves ou irréversibles, l'absence de certitude scientifique absolue ne doit pas servir de prétexte pour remettre à plus tard l'adoption de mesures effectives visant à prévenir la dégradation de l'environnement ". Réaffirmation de ce principe, en juin 1997, à New York, dans une approche " développement durable ", à l'occasion de la déclaration finale du deuxième Sommet de la Terre.

\subsection{Sur le plan national}

La France est un des premiers Etats à avoir intégré ce principe dans son droit interne avec la Loi du 13 juillet 1992 relative au contrôle et à l'utilisation des organismes génétiquement modifiés -OGM- (qui organise un régime d'autori- sation préalable, notamment au regard des effets irréversibles potentiels des OGM). Puis la Loi française du 2 février 1995, dite Loi Barnier, sur le renforcement de la protection de l'environnement, le formule en ces termes : "l'absence de certitudes, compte tenu des connaissances scientifiques du moment, ne doit pas retarder l'adoption de mesures effectives et proportionnées visant à prévenir un risque de dommages graves et irréversibles à l'environnement à un coût économiquement acceptable ".

Aujourd'hui, le principe de précaution dépasse largement le domaine de l'environnement : la protection de la santé de l'homme est, en effet, un de ses objectifs premiers. Ce principe a, par exemple, été évoqué dans l'affaire de l'hormone de croissance, dans la polémique sur la transmission à l'homme de l'encéphalopathie spongiforme bovine (maladie de la vache folle) ou encore, très récemment, concernant la mise sur le marché du maïs transgénique.

\section{PRINCIPE DE BLOCAGE OU PRINCIPE DE PROGRÈS ?}

D'un côté : interprétation radicale de la précaution (problématique du " risque zéro ") qui privilégie une logique d'inaction consistant à préférer, selon l'adage " dans le doute, abstiens-toi ", l'impossibilité d'engagement, la réduction ou la cessation totale de certaines activités industrielles tant que l'on ignore les conséquences, ou de façon plus restrictive encore, tant que l'on n'a pas fait la preuve de l'innocuité absolue des activités. A l'autre extrême, l'interprétation radicale de la précaution peut conduire à favoriser une logique d'action forcée consistant à imposer des mesures visant à modifier des situations qui font seulement craindre un danger.

De l'autre : interprétation modérée de la précaution que fait prévaloir la loi Barnier, tout comme la Déclaration de Rio de 1992, privilégiant une logique d'action prudente sur toute règle d'abstention ou d'action forcée. La mise en 
œuvre du principe de précaution repose alors sur deux critères principaux :

- la démarche " de précaution" doit être entreprise chaque fois que l'activité comporte un risque de dommages graves et irréversibles : adoption de mesures de précaution effectives et proportionnées par rapport à la prise de risque dans le cadre de l'activité à engager ou en cours.

- la démarche doit conduire à accompagner l'activité considérée de mesures de protection dont l'une des limites est l'acceptabilité économique de leur coût.

\section{III - L'INCERTITUDE SCIENTIFIQUE : FACTEUR CLÉ DE LA PRÉCAUTION}

On l'a vu : au sens de la Loi Barnier, le principe de précaution doit avant tout être perçu comme un véritable état d'esprit permettant de traiter les problèmes complexes liés à la gestion des risques incertains, et ce de façon transparente et cohérente.

L'essentiel de ces problèmes concerne la toxicité chronique provoquée par de faibles doses d'exposition répétées dans le temps (ex. : champs électromagnétiques de basse fréquence, faibles doses de rayonnements ionisants liées aux fonctionnement des installations nucléaires, rejets et déchets contenant de faibles quantités de substances chimiques toxiques...). Les risques sont alors plus difficiles à objectiver pour des raisons méthodologiques : l'intensité des effets est faible ; la caractérisation de l'exposition est délicate quand celle-ci est constituée d'un mélange complexe de contaminants à faible concentration ; les signes apparaissent après un délai de latence qui peut atteindre plusieurs dizaines d'années pour des maladies comme le cancer; les maladies provoquées par ces contaminants ne sont pas spécifiques ; elles sont multifactorielles. Bref, dans ce contexte, l'affirmation d'une relation de causalité prête souvent à discussion pour ne pas dire à spéculation.

L'incertitude scientifique annonce la faillite de la norme et l'ébranlement de la confiance. Or, dans la gestion de situations incertaines, la confiance est un paramètre clé sans lequel le décideur est paralysé. Son dilemme est de trouver alors un juste équilibre entre 2 attitudes : la sous-réaction qui engagerait sa responsabilité si le danger finissait par être démontré ; la sur-réaction, qui conduirait à inquiéter inutilement la population et à gaspiller des ressources dont d'autres problèmes auraient pu bénéficier.

Chacun s'accorde désormais sur le fait que l'incertitude ne peut plus justifier l'inaction ; le caractère insidieux de l'exposition, le fait qu'elle soit inégalement répartie et surtout l'incertitude des effets à long terme, tout concourt à ce que le risque soit surestimé. D'autant que les outils traditionnels de gestion du risque sont inopérants (du style : si quelque chose est jugé dangereux, on l'interdit - amiante, peinture au plomb - ou on en limite l'exposition - rayonnements ionisants, benzène...-). Prenons, en effet, le cas des champs électro-magnétiques. Les interdire reviendrait à arrêter la production de l'énergie électrique. Quant à en limiter l'exposition, il faudrait pour cela que les données scientifiques permettent de tracer une relation dose-effet, ce qui n'est pas le cas.

Le principe de précaution traduit alors le dilemme qui se pose entre l'incertitude scientifique et la nécessité de prendre des décisions dans un tel contexte de doute. II y a nécessité alors de quitter le domaine de la science pour se tourner vers l'éthique de l'action en adoptant une attitude prudente permettant d'éviter de créer une situation d'irréversibilité. Adopter une telle attitude prudente conduit, pour le décideur, à un questionnement permanent qui lui permettra d'entrer alors dans une problématique de réduction du risque.

Qu'est-ce qu'être responsable dans l'action en univers incertain?

Dans un tel contexte d'incertitude, comment organiser la prise de risque au niveau social et donc son acceptabilité ?

\section{IV — L'APPLICATION DU PRINCIPE DE PRÉCAUTION}

Si la précaution recommande d'agir de façon prudente, elle n'indique pas comment construire l'action pour que le risque incertain soit socialement acceptable. Ce qui revient à se demander comment va s'organiser la prise et le partage de ce risque au niveau social ?

Pour générer l'acceptabilité sociale de ce risque incertain. il faut une organisation sur la base du volontariat et de la responsabilisation des acteurs et ce au niveau de la mise en cuvre de la vigilance. En d'autres termes, l'application du principe de précaution nécessite une approche nouvelle du processus décisionnel.

En premier lieu, intégrer à la décision les risques incertains (qu'il faut donc anticiper) et envisager les moyens de les réduire. Ainsi, les effets dommageables d'une décision seront-ils restreints par avance. De même, la pertinence des actions, dès les premiers signaux d'alerte, sera-t-elle garantie (cette approche est inverse à celle traditionnelle fondée sur la réparation).

C'est dans l'objectif de faire face aux craintes et aux incertitudes qu'a été créé, par arrêté du M.A.T.E. du 30/07/1996, le Comité de Prévention et de la Précaution (à la suite des affaires fortement médiatiques de l'ESB, de l'amiante, des hormones de croissance), afin que soit organisée en France, dans le cadre d'un dispositif interministériel, une expertise collective de veille et d'alerte indépendante et multiple, sur l'ensemble des questions d'environnement susceptibles d'avoir des incidences sur la santé humaine (qualité de l'air, de l'eau, des sols, des rayonnements, du bruit et des substances chimiques et biologiques).

En second lieu, approche raisonnable sur le plan économique en ce qui concerne les moyens alloués à la protection, ce afin de rester dans l'interprétation modérée du principe voulue par le législateur.

En $3^{\text {ème }}$ lieu, approche évolutive qui prenne en compte l'évolution du contexte scientifique, technique et économique.

Enfin, dans l'élaboration des choix, recherche permanente du dialogue, de la transparence à l'égard des informations disponibles, ainsi qu'acceptation de débattre de son expertise.

Une telle démarche a été mise en œuvre en radioprotection avec le principe ALARA (as low as reasonnably achievable, aussi bas que raisonnablement possible) qui traduit le niveau particulier et non plus social de la prudence en organisant au cas par cas des attitudes propres à réduire le risque radiologique, aussi bas que raisonnablement possible, compte tenu des facteurs économiques et sociaux. Le principe ALARA exprime la figure aboutie et qualitative de la prudence. 
En bref: " Le plus inacceptable n'est pas que le décideur se trompe alors qu'il fait face à l'incertain, mais que sa démarche décisionnelle ne soit ni transparente, ni cohérente, ni explicite. Il est désormais crucial d'expliciter les critères quantitatifs et qualitatifs utilisés pour asseoir les décisions, organiser les débats et accroître leur lisibilité " (Dr Dab, Sce des Etudes Médicales EDF)

\section{Q QUALIFICATION JURIDIQUE DU PRINCIPE DE PRÉCAUTION}

Le statut qui sera reconnu au principe de précaution oscille entre d'une part celui de simple principe politique orientant l'action des décideurs et d'autre part, celui de norme légale qui sera alors prise en compte par les juges dans l'évaluation des intérêts concurrents.

L'interprétation modérée de ce principe l'assimile à une obligation de moyens ou de comportement qui incite chacun à multiplier les précautions face au risque incertain (aucun résultat défini a priori ne saurait être exigible puisqu'aucun n'est sûr).

A l'opposé, pour les tenants de l'interprétation radicale du principe de précaution, celui-ci s'analyse en une véritable obligation de résultat, ce qui les conduit, selon I'adage "dans le doute, abstiens-toi ", à exiger un moratoire sur les actions ou activités industrielles. Certains réclament ainsi que l'on réduise ou que l'on cesse certaines d'entre elles au motif qu'elles engendrent une incertitude scientifique, bien qu'il n'ait pas été démontré qu'elles aient des conséquences préjudiciables pour la santé.

Le Conseil d'Etat (sur recours de Greenpeace/Ecoropa) a suspendu - au nom du principe de précaution -, le 25 septembre dernier, l'autorisation, par le ministère de l'agriculture, de mise en culture du maïs transgénique de la firme Novartis. Pour ce faire, il n'a pas suivi les conclusions du Commissaire du Gouvernement qui considérait qu'il était juridiquement difficile d'invoquer le principe de précaution qui, tel que formulé par la Loi Barnier, n’a, selon lui, aucune valeur juridique contraignante.

\section{Références Bibliographiques}

[1] BOEHLER M-C. Le Principe de Précaution - " ENERGIES-SANTE " 1997.

[2] BOEHLER M-C. Le Principe de Précaution et la Radioprotection " Radioprotection et Droit Nucléaire ", collection Stratégies énergétiques et Société, Genève 1998.

[3] BOEHLER M-C. A propos du Principe d'optimisation de la protection radiologique et du principe de précaution, Revue Générale Nucléaire Nº/Novembre-Décembre 1996.

[4] Docteur William DAB, Le Principe de Précaution et l'exemple des Champs Electromagnétiques - Aménagement et Nature $\mathrm{N}^{\circ} 118$. 